\title{
Infodemiology and Infoveillance of Multiple Sclerosis in Italy
}

\author{
Nicola Luigi Bragazzi \\ School of Public Health, Department of Health Sciences (DISSAL), University of Genoa, Via Antonio Pastore 1, 16132 Genoa, Italy \\ Correspondence should be addressed to Nicola Luigi Bragazzi; robertobragazzi@gmail.com
}

Received 10 February 2013; Revised 2 June 2013; Accepted 3 June 2013

Academic Editor: Francesco Patti

Copyright (C) 2013 Nicola Luigi Bragazzi. This is an open access article distributed under the Creative Commons Attribution License, which permits unrestricted use, distribution, and reproduction in any medium, provided the original work is properly cited.

\begin{abstract}
Multiple Sclerosis (MS) is a chronic debilitating disease of probable autoimmune inflammatory nature, whose aetiology is not fully understood, despite the many efforts and investigations. In this manuscript, we review the concept of "Multiple Sclerosis 2.0", that is to say the Internet usage by MS patients, for seeking health and disease-related material for self-care and self-management purposes, and we introduce a Google Trends-based approach for monitoring MS-related Google queries and searches, called MS infodemiology and infoveillance. Google Trends has already proven to be reliable for infectious diseases monitoring, and here we extend its application and potentiality in the field of neurological disorders.
\end{abstract}

\section{Introduction}

1.1. Multiple Sclerosis 2.0. Multiple Sclerosis (MS) is a chronic debilitating disease of probable autoimmune inflammatory nature, whose aetiology is not fully understood, despite the many efforts and investigations carried out $[1,2]$. Being a chronic disorder, MS has a tremendous psycho-social burden $[3,4]$, and recently the concept of a Web-based aid for MS patients has emerged, collecting MS-related information and at the same time trying to reduce the stressors, enhancing the self-management of the disease, facilitating the interactions between the patients and the medical team, and accurately reporting to the physician the patients' symptoms after their online registration [5].

Gunther Eysenbach coined the terms "infodemiology" and "infoveillance", describing a new emerging approach for public health [6,7], based on large-scale monitoring and data mining, within the conceptual framework of e-health and health Web $2.0[8,9]$. Even if with some limitations and concerns, the Internet and the medical informatics are paving the way for new directions in the field of the epidemiological research, indicating new trends and strategies [10]. In the shift from a paternalistic medicine (P0 model) to a patient-centered approach (P6 model, where the six Ps stay personalized, preventive, predictive, participatory, psycho cognitive, and public) [11-13], patients tend to use Internet as a source of relevant health-related information, even if not all properly validated or reliable, for health education, for finding suggestions, for coping strategies, and for self-managing their disease [14]. The Internet has blurred geographical boundaries and other barriers, making it available to lay people a wealth of medical material which was rather difficult to reach before that [15]. This material could help the patients in the process of decision making, providing understandable, clear information, and decreasing their anxiety level [15]. Patients are attracted by the possibility not only to access medical material, but also to upload and create their own contents (generally known as User-Generated Content (UGC), User-Driven Content (UDC), in specific cases termed as Patient-Generated Content (PGC)), and this is opening new unprecedented avenues and scenarios, which were before unforeseeable [14, 16]. New forms and ways of communication have modified the patient-physician relationship and have introduced the so-called "Patient 2.0 phenomenon" [17].

In the "Multiple Sclerosis 2.0" era, many MS patients would accept or already regularly make use of innovative Web technologies and electronic forms of communication, as reported by Haase and collaborators [18]. The number of MS patients who use YouTube to share their experiences, to seek for advice, and to evaluate and comment on other users videos is increasing. Fernandez-Luque and collaborators [19] found that MS patients using YouTube were surprisingly informed about the latest drugs against MS. Patients share their health-related status using platforms like "PatientsLike" 
and other forum/blogs, and this behavior has proven to result in better clinical outcomes [20, 21]. Atreja and coworkers [22] found that MS patients judged valuable and of vital importance for them to access the Internet for MS-related information. These findings confirmed the results obtained by Hay and collaborators [23], who reported that most MS patients consulted the Web before and after the medical visit, in order to better understand the technical terms used by the physicians. They should be aware of this aspect and try to use a more patient-friendly vocabulary, and they should know which material is available on the Web, in order to react to it by providing further information or correcting some mistakes, and to discuss it with the patient. On the other hand, patients themselves are not likely to report the Internet materials to the doctors, and some of them fear that doing so would mean a lack of trust in the physicians' skill and professional competence or a challenge to their authorities [24]. Other patients experience a lack of time or time burden and constraints during the visits [15]. Most patients, indeed, do not frequently bring the information surfed on the Internet to their clinic visits, that is to say sharing that material with their doctors or asking them questions based on the Web searches [15]. Moreover, doctors rarely advice their patients to search health-related material on the Internet [15]. Lejbkowicz and collaborators [25] found that most MS patients consult online information and that the Internet usage positively correlates with the MS status, severity, and degree of disability. Moreover, they consider online information as reliable but more accessible and understandable than the book and manual materials, and thus beneficial, without harmful effects. Marrie and coworkers [26] found similar results, and using a logistic regression model and younger age, less degree of disability, higher annual earning were predictor of the Internet usage. Therefore, neurologists should interact with the patients and provide them with a detached report of the effective quality of the Web-based material and refer them to specific websites, as the Information $\mathrm{Rx}$ project (http://www.medlineplus.com/) - a joint venture from the American College of Physicians and the National Library of Medicine (NLM) - recommends to explore different and new information channels. To better discuss with MS patients, doctors should know which are the most frequent hit searches and their needs or knowledge gaps [27]. For this purpose, Google Trends could play a major role.

1.2. Google Trends. Google Trends is a Web application that enables to visualize hit searches volumes [28]. Indeed, the Google-based approach has recently emerged as a new tool in the field of infodemiology and infoveillance [29]. It is particularly useful to monitor especially influenza epidemics [30-33]. SARS was discovered through monitoring search engines, since Larry Brilliant discovered a "viral" search of anti-influenza drugs in China [34].

Scholars have stressed the correlations between Internet search and the triage data, the hospital access, and the need of drugs [35,36].
Google Trends has been usually used for monitoring infectious diseases (from influenza to tuberculosis and other emerging or drug-resistant infectious strains) [37, 38], but its enormous potentiality and applications in other fields of medicine have been noticed only recently. Gunn III and Lester surveyed suicides using search engines [39], and other scholars have replicated these findings [40, 41], while Deluca and collaborators exploited the Web for studying the epidemiology of drug addiction [42]. Internet key-word searching has been used also in the field of human sexuality, trying to better understand the dating and mating behaviors $[43,44]$. another interesting application of Web hit search is the real-time detection of kidney stone diseases [45], but so far, to our knowledge, no one has explored the possibility of monitoring Google searches that refer to MS and associated concepts.

\section{Materials and Methods}

2.1. Google Trends. Google Trends, an online tracking system of Internet hit search volumes, recently merged with its sister project Google Insights for Search, which was accessed from 2004 to 2012, since data before 2004 were not available on searching for "sclerosi multipla" (Italian for MS) [28]. All the queries have been downloaded and analyzed. It is noteworthy to remember that Google Trends provides the user data that are scaled and normalized in such a way that the numbers reflect how many searches have been performed for a particular term or category, relative to the total number of queries carried out on Google over time, rather than representing absolute search volume numbers. For this reason, data are analyzed as NFV (normalized flux volume) or NSV (normalized search volume). Moreover, in order to avoid any bias in treating and manipulating the data, these have been rescaled and renormalized, taking into account the Digital Divide (North-South gradient in the access to the Internet and different Internet usages over the time). Corrections have been applied, using data from the ISTAT (Italian National Institute of Statistics). This has ensured robust findings.

2.2. Statistical Analysis. Autocorrelation is the correlation of a parameter with itself over the time; autocorrelation functions of this series of data, both crude and adjusted partial, were computed using $\mathrm{R}$ environment [46]. The wavelet transform of the time series and the wavelet power spectrum (WPS) analysis were carried out using algorithms written in Matlab accessible on http://paos.colorado.edu/ research/wavelets/ [47, 48]. The multiple linear regression fitting was performed in R environment [49]. A list of MS symptoms and MS-related terms was searched in Italian language and their flux volumes were correlated with the MS hit search data. Pearson's correlation coefficient, that is to say the measure of the linear correlation between two variables, was calculated with SPSS software V21.0.0 package (IBM) and using R environment [50]. $P$-values were computed with SPSS, and values equal or less than 0.05 were considered statistically significant. 


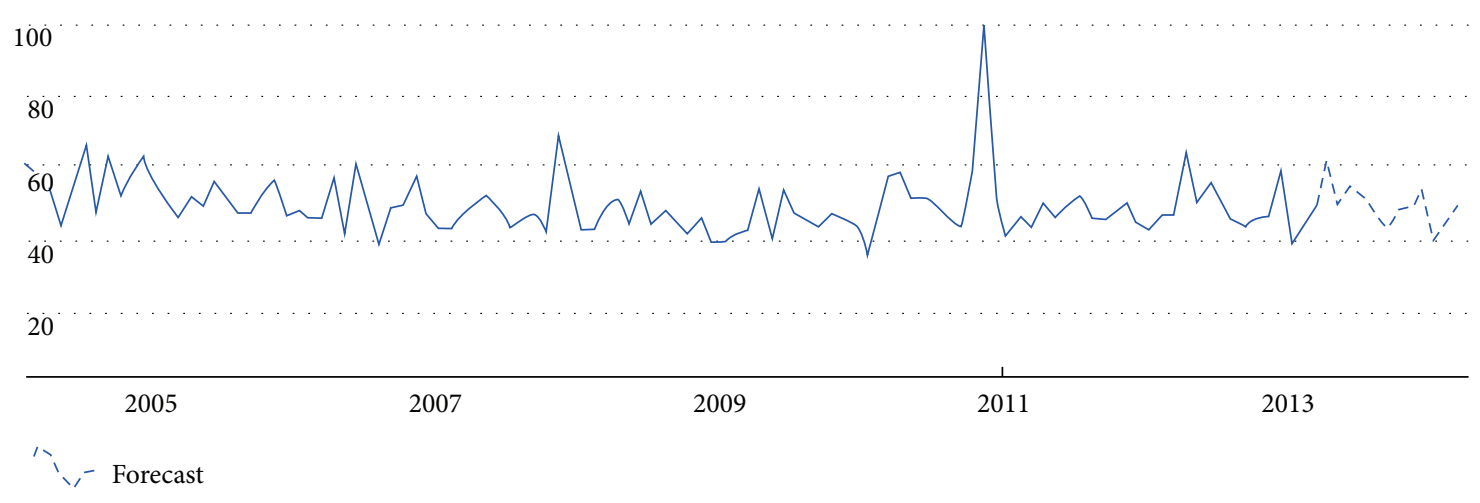

(a)

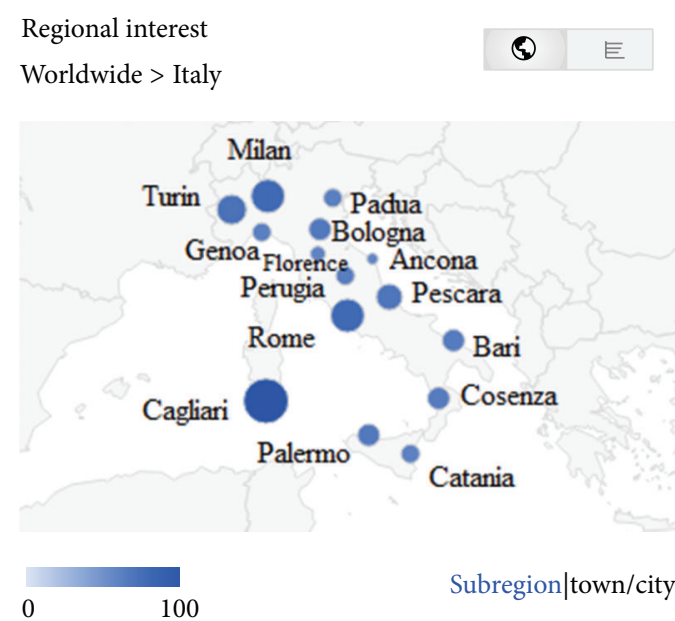

(b)

\begin{tabular}{|c|c|c|}
\hline Related terms & Top & Rising \\
\hline Sclerosi multipla sintomi & 100 & \\
\hline La sclerosi multipla & $30 \rightleftharpoons$ & \\
\hline Sclerosi multipla zamboni & 15 - & \\
\hline Zamboni & $15=$ & \\
\hline Sla & $10=$ & \\
\hline Sclerosi multipla cura & $10=$ & \\
\hline Associazione sclerosi multipla & $10=$ & \\
\hline Cure sclerosi multipla & $10=$ & \\
\hline Diagnosi sclerosi multipla & $10=$ & \\
\hline Cause sclerosi multipla & $10=$ & \\
\hline
\end{tabular}

(c)

Figure 1: Google Trends-based MS hit search volume over the years from 2004 to 2012 and forecast trend projected over 2013 (a), a map of labeled Italian towns with higher flux volumes (b), and a list of the most searched MS-related terms (c).

\section{Results}

3.1. MS Time Series. The pattern of Internet search volume (Figure 1) did not reveal a cyclic trend, as can be seen from the autocorrelation diagram (Figure 2). No annual or seasonal trends were found. The flux volume remained constant from 2004 to 2012, apart from a peak in 2007-2008 and another one in 2011-2012. However, multiannual (4-5 years) long-term trends were revealed by the exploratory analysis carried out with the WPS technique (Figure 3 ) and confirmed by the multiple linear regression $(P$ value 0.023 , intercept 28.76 , sine-regression coefficient 1.07 , and cosine-regression coefficient -0.73 ) (Figures $4(\mathrm{a})$ and $4(\mathrm{~b})$ ).

3.2. MS-Related Google Trends Queries. MS therapy and symptoms are the most searched MS-related terms by the users. In most cases, the Pearson's correlation coefficients yielded a statistical significance $(P$-value $<0.05)$ (Figure 5 , Table 2). Some of these correlations have a positive sign, like MS and sexual dysfunctions, MS and depression (for the category of MS and symptoms), MS and Zamboni's intervention, MS and stem cells (for the category of MS and treatments/therapy), while others a negative one (viz., MS and interferon for the category of MS and treatments/therapy; MS and paresthesia; MS and dysphagia; MS and ophthalmologic symptoms; MS and gastrointestinal symptoms for the category of MS and symptoms).

\section{Discussion}

4.1. MS Time Series. The main finding of this article is the feasibility of adopting a Google Trends-based survey for MS. The results have been compared with those present in the literature (about epidemiology, both temporal and spatial, and the Internet usage) and have been validated.

Two main peaks can be observed in the time series. The first one is related to the public disclosure of Nicoletta Mantovani of being suffering from MS. The second observed peak may be correlated with the introduction of Paolo Zamboni's therapy, which attracted a lot of media and layed public 


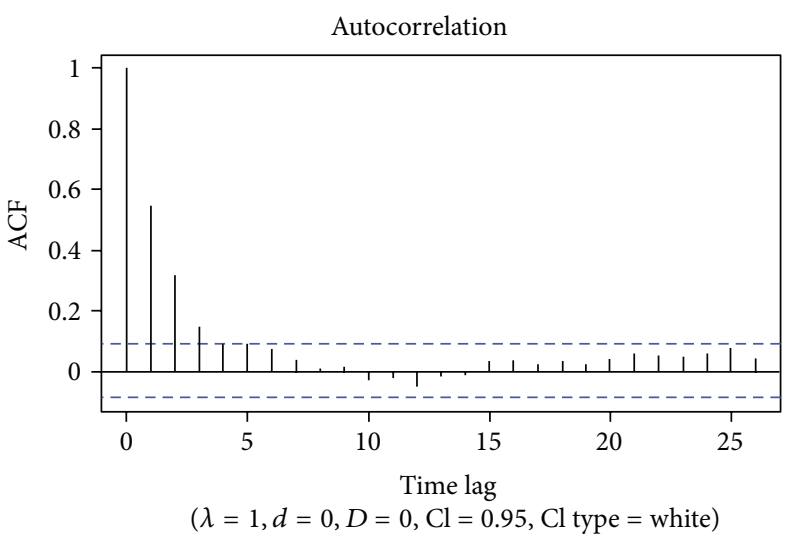

(a)

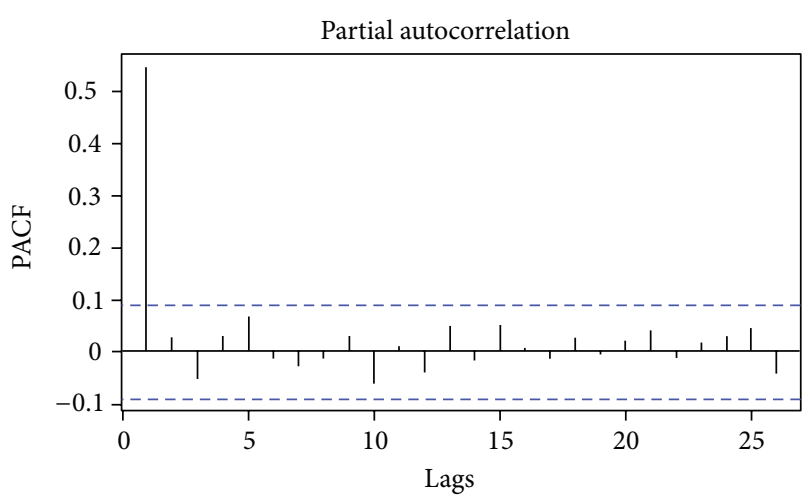

$(\lambda=1, d=0, D=0, \mathrm{Cl}=0.95, \mathrm{Cl}$ type $=$ white $)$

(b)

FIGURE 2: Autocorrelation plot for the MS hit search (a) and partial autocorrelation plot (b), showing no annual cyclical pattern or regular trend.

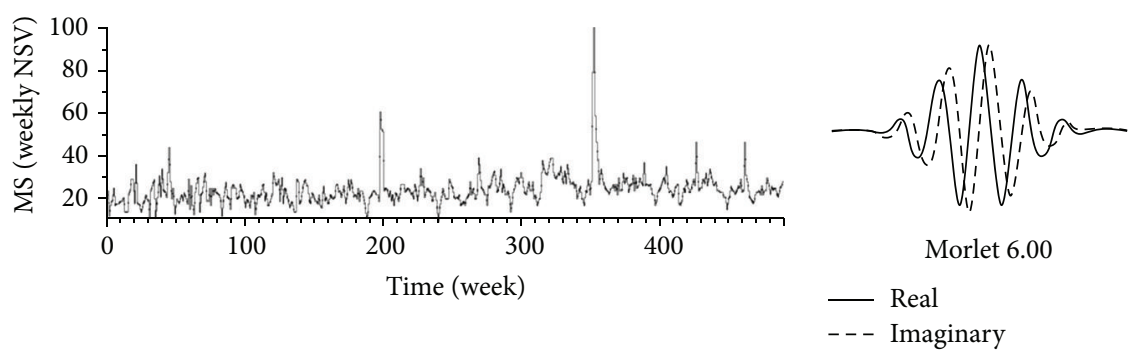

(a)

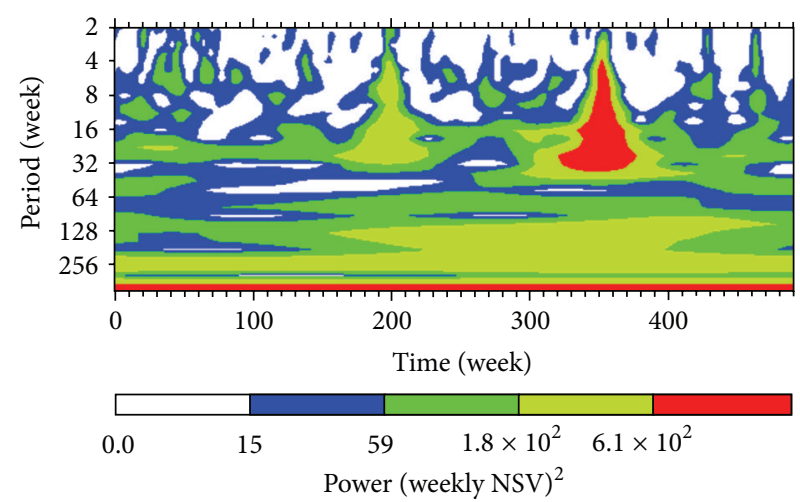

(b)

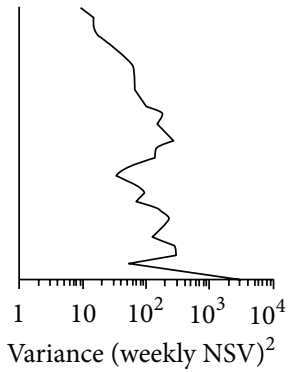

(c)

FIGURE 3: Exploratory spectral analysis, showing (a) MS time series and (b) the wavelet power spectrum (WPS) analysis. The contour levels are chosen so that $75 \%, 50 \%, 25 \%$, and $5 \%$ of the wavelet power are above each level, respectively. (c) The global wavelet power spectrum (GWPS) analysis. These analysis were carried out using algorithms written in Matlab and accessible on http://paos.colorado.edu/research/wavelets/. Time unit is week; NSV stays for normalized search volume.

interest. Paolo Zamboni's therapy is a controversial surgical operation, devoted to cure the Chronic Cerebrospinal Venous Insufficiency (CCSVI), a new nosological entity which Zamboni suggested to be the cause or one of the causes of MS in a high percentage of MS patients [51]. As noticed by Vera et al. [52] and by Machan and collaborators [53], the number of online resources and material related to CCSVI is increasing, and peer groups are discussing this opportunity on social networks and dedicated forums. Nicoletta Mantovani herself announced she was healed after undergoing Zamboni's operation. Apart from these peaks, that can be considered as transitory bursts, the search volume remained quite constant. Other studies have validated this finding, confirming that media interests do not perturb time series which are rather robust (see, e.g., [54] and references therein).

It is controversial whether the prevalence and the incidence of MS have remained unvaried or increased in the last years, since some studies report an observed increase [5558], while other fail to replicate these findings [59]. Some 


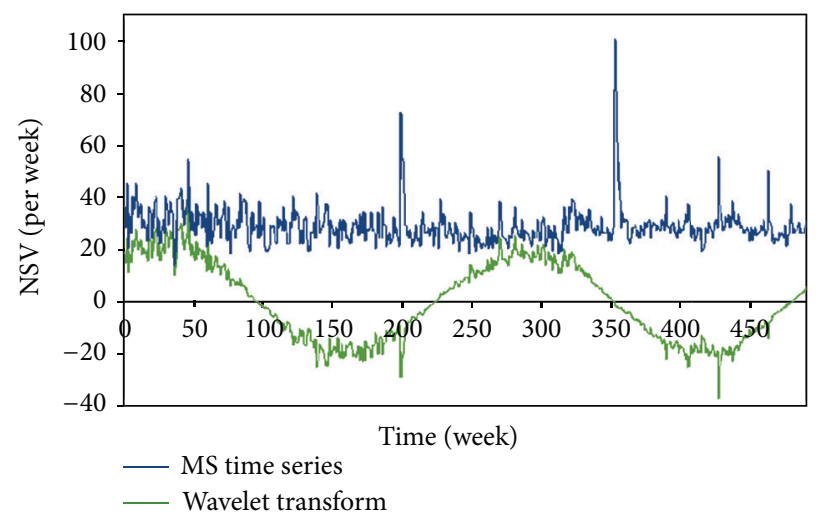

(a)

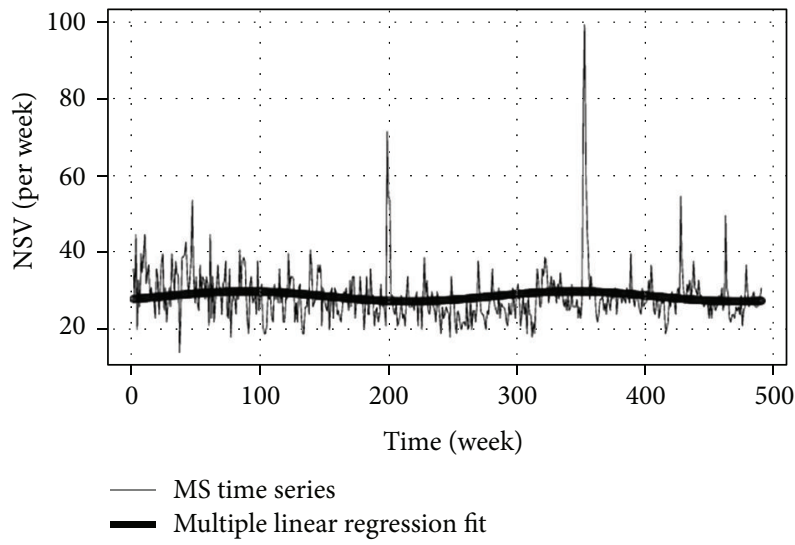

(b)

FIgURE 4: (a) The wavelet transform of the MS time series data. (b) The multiple linear regression fit of the MS time series data.

TABLE 1: A list of the towns (first column) with the higher normalized MS hit search flux volumes (NFV), as provided by Google Trends. In the third column, these crude NFV data have been rescaled taking into account the Digital Divide and North-South access to the Internet (according to the ISTAT) and re-normalized. We term these data the adjusted NFV.

\begin{tabular}{lcc}
\hline City/town & Crude NFV (\%) & Adjusted NFV (\%) \\
\hline Cagliari & 100 & 100 \\
Rome & 80 & 93 \\
Milan & 80 & 93 \\
Turin & 74 & 86 \\
Pescara & 71 & 82 \\
Palermo & 69 & 66 \\
Bologna & 69 & 80 \\
Bari & 67 & 64 \\
Cosenza & 67 & 64 \\
Perugia & 66 & 77 \\
Padua & 65 & 75 \\
Genoa & 65 & 75 \\
Catania & 64 & 61 \\
Florence & 62 & 72 \\
Ancona & 60 & 70 \\
\hline
\end{tabular}

scholars have argued that the increase could be explained taking into account environmental factors, while others have stated that it is only an apparent increase which may be due to confounding biases, better diagnostic criteria and therefore better case ascertainment, and longer survival rates as well as newly introduced diagnostic criteria and definitions, incorporating more sophisticated and particularly sensitive paraclinical and preclinical tools, and availability and accessibility of qualified structures and personnel [60]. However, Google Trends data were not available before 2004 and we failed to investigate MS-related queries over a longer range.

It is interesting to notice that the Italian towns with the higher searching fluxes and volumes include Cagliari, and
TABle 2: Pearson's correlation coefficients for different hit search volumes related to MS and their statistical significance according to their two tails $P$ value.

\begin{tabular}{lcc}
\hline Hit search volume & $\begin{array}{c}\text { Pearson's } \\
\text { correlation } \\
\text { coefficient }\end{array}$ & $\begin{array}{c}P \text { value } \\
\text { (two-tails) }\end{array}$ \\
\hline MS and symptoms & $r=0.02$ & $P=0.66$ \\
MS and depression & $r=0.11$ & $P=0.02^{*}$ \\
MS and anxiety & $r=-0.04$ & $P=0.44$ \\
MS and paresthesia & $r=-0.11$ & $P=0.01^{*}$ \\
MS and dysphagia & $r=-0.10$ & $P=0.03^{*}$ \\
Ophthalmologic manifestations of MS & $r=-0.12$ & $P=0.01^{*}$ \\
MS and dysarthria & $r=-0.00$ & $P=0.96$ \\
MS and bladder problems & $r=0.04$ & $P=0.36$ \\
MS and gastrointestinal problems & $r=-0.14$ & $P=0.00^{*}$ \\
MS and sexual dysfunction & $r=0.10$ & $P=0.03^{*}$ \\
\hline \multicolumn{1}{c}{ MS and treatments/therapy } & \\
MS and Paolo Zamboni's therapy & $r=0.35$ & $P=0.00^{*}$ \\
MS and stem cells & $r=0.12$ & $P=0.01^{*}$ \\
MS and methotrexate & $r=-0.09$ & $P=0.05$ \\
MS and corticosteroids & $r=-0.07$ & $P=0.15$ \\
MS and interferon & $r=-0.10$ & $P=0.04^{*}$ \\
\hline
\end{tabular}

${ }^{*}$ Statistically significant.

Sardinia is a well-known high risk area [61, 62] (Figure 1, Table 1). The other towns are equally spatially distributed; that is to say we do not observe any geographical gradient between North and South in terms of Google Trends query and search fluxes and volumes, as confirmed by many detailed epidemiological Italian surveys, which have proven to be against the "latitude theory" introduced by Millefiorini et al. [63]. However, it must be noticed that some scholars while criticizing the latitudinal gradient theory for incidence maintain that the latitudinal gradient theory for prevalence may be still valid [64]. 


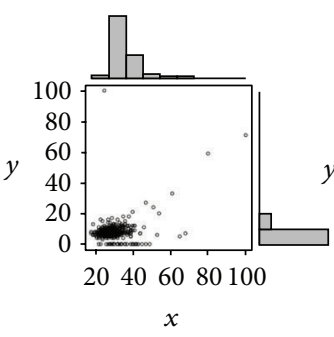

MS and Paolo Zamboni's therapy

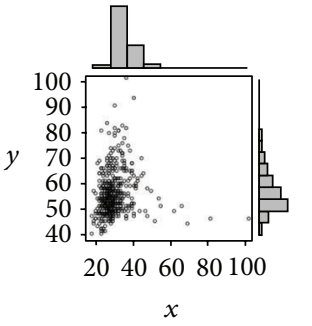

MS and depression

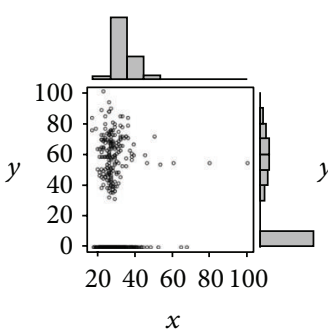

MS and dysarthria

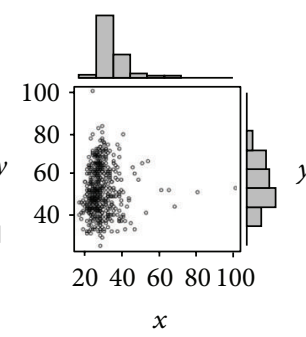

MS and fatigue

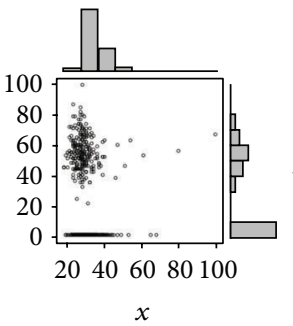

MS and methotrexate

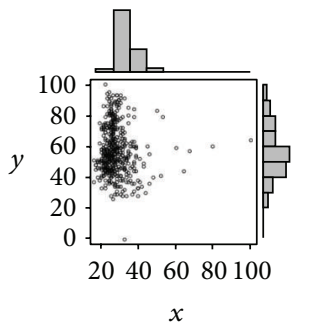

MS and corticosteroids

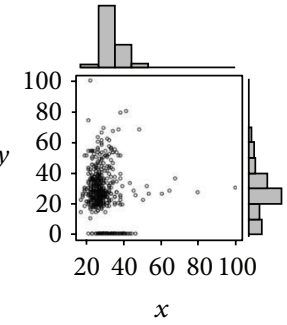

MS and interferon
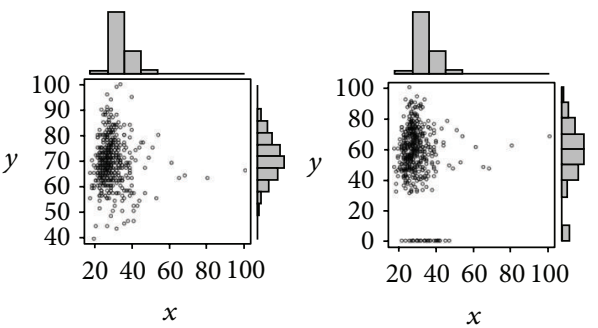

MS and paresthesia

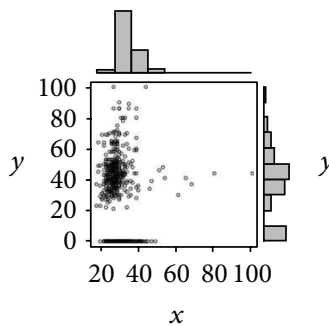

MS and dysphagia

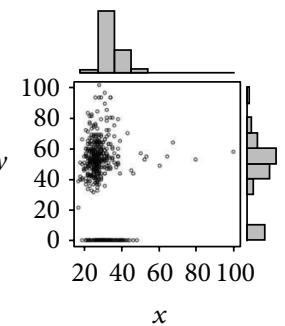

Ophthalmologic manifestations of MS

FIGURE 5: Pearson's correlation plots for some of the most important MS-related hit search terms.

4.2. MS Google Trends Queries. We divided the most search terms in two categories, namely, and MS and symptoms, MS and treatments/therapy. This finding confirms a research carried out by Lejbkowicz and collaborators [25] that "understanding the disease" and "treatments" were the most viewed topics. Even though statistically significant, each MS-related term has a low Pearson's correlation coefficient, and for this reason only a panel of several words reach a good overall coefficient. This suggests that MS patients would not be limited to search for a few words, but for an array of terms. The different signs (minus and plus) of the correlations may reflect a different time pattern of MS-related queries and could help the clinicians in better understanding the patients' needs and requests.

4.3. Limitations. However, this study has some limitations. For example, we monitored the trend of the Internet searches volumes over the time, but we did not investigate the completeness nor the reliability of the Web material related to MS. For these reasons, this study should be complemented with a content analysis of the Web sites and with ad hoc surveys to MS patients, asking them their understanding and their reaction to the online material and information, as well as their Internet usage. Actually, this is a serious gap in the current Italian research. It would be clinically important to design specific Web content, in order to meet the patients needs, requests, and expectations. Moreover, this could pave the way for interventions made directly by the practitioners to interact with the patients, to post high-quality material dedicated to MS symptoms and treatments, and answer patients' doubts and questions. Lowe-Strong and McCullagh [65] implemented a Web Portal endowed with a user-friendly visual interface for pain and MS-related symptoms selfrecording, while other sites enabling the self-monitoring of MS symptoms, prescriptions orders, laboratory and clinical results retrieval, online patient education, news and updates on MS research, and a timely communication with the medical staff are being designed [66]. Recently, Jones and coworkers have created a dynamic interactive Web Portal, where MS patients can record their symptoms and the quality of life [67]. Boeschoten and collaborators [68] developed a Webbased problem-solving treatment (PST) specifically designed for MS patients suffering from depressive symptoms.

\section{Conclusion}

In this contribution, we showed that a Google Trends-based infodemiology and infoveillance system could be successfully 
applied also to monitor MS-related information and Google queries and probably to other chronic degenerative disorders, and not only to infectious diseases. Even with its warranted limitations, Web-based epidemiology has a promising potentiality.

\section{References}

[1] M. J. Tullman, "Overview of the epidemiology, diagnosis, and disease progression associated with multiple sclerosis," American Journal of Managed Care, vol. 19, supplement 2, pp. S15-S20, 2013.

[2] I. Bordi, I. R. Umeton, V. A. Ricigliano et al., "A mechanistic, stochastic model helps understand multiple sclerosis course and pathogenesis," International Journal of Genomics, vol. 2013, Article ID 910321, 10 pages, 2013.

[3] N. L. Bragazzi, "The gap in the current research on the link between health locus of control and multiple sclerosis: lessons and insights from a systematic review," Multiple Sclerosis International, vol. 2013, Article ID 972471, 8 pages, 2013.

[4] G. Del Puente, A. Mahamid, and N. L. Bragazzi, "Health locus of control and multiple sclerosis: a systematic review," Journal of Psychology and Psychotherapy, vol. S3, p. 001, 2012.

[5] D. V. Ford, K. H. Jones, R. M. Middleton et al., "The feasibility of collecting information from people with Multiple Sclerosis for the UK MS Register via a web portal: characterising a cohort of people with MS," BMC Medical Informatics and Decision Making, vol. 12, p. 73, 2012.

[6] G. Eysenbach, "Infodemiology and infoveillance: tracking online health information and cyberbehavior for public health," American Journal of Preventive Medicine, vol. 40, no. 5, pp. S154S158, 2011.

[7] G. Eysenbach, "Infodemiology and infoveillance: framework for an emerging set of public health informatics methods to analyze search, communication and publication behavior on the Internet," Journal of Medical Internet Research, vol. 11, no. 1, p. ell, 2009.

[8] T. M. Burkow, L. K. Vognild, T. Krogstad et al., "An easy to use and affordable home-based personal eHealth system for chronic disease management based on free open source software," in Studies in Health Technology and Informatics, vol. 136, pp. 8388, IOS Press, 2008.

[9] D. Giustini, "How Web 2.0 is changing medicine," British Medical Journal, vol. 333, no. 7582, pp. 1283-1284, 2006.

[10] B. K. Lee, "Epidemiologic research and Web 2.0-the user-driven Web," Epidemiology, vol. 21, no. 6, pp. 760-763, 2010.

[11] N. L. Bragazzi, "From P0 to P6 Medicine, a model of highly participatory, narrative, interactive and "augmented" medicine: some considerations on Salvatore Iaconesi's clinical history," Patient Preference and Adherence, no. 7, pp. 353-359, 2013.

[12] N. L. Bragazzi, "Children, adolescent and young adults participatory medicine: involving them in the health care process as a strategy for facing infertility issue," American Journal of Bioethics, vol. 13, no. 3, 2013.

[13] N. L. Bragazzi and G. Del Puente, "Why P6 medicine needs clinical psychology and a trans-cultural approach," Health Psychology Research, vol. 1, no. 1, p. e5, 2013.

[14] A. Burton, "YouTube-ing your way to neurological knowledge," The Lancet Neurology, vol. 7, no. 12, pp. 1086-1087, 2008.

[15] D. Blanch, C. Sciamanna, H. Lawless, and J. Diaz, "Effect of the internet on the doctor-patient relationship: a review of the literature," Journal on Information Technology in Healthcare, vol. 3, no. 3, pp. 179-201, 2005.

[16] J. Y. Kim, T. M. Gudewicz, A. S. Dighe, and J. R. Gilbertson, “The pathology informatics curriculum wiki: harnessing the power of user-generated content," Journal of Pathology Informatics, no. 1, p. 10, 2010.

[17] L. Bos, A. Marsh, D. Carroll, S. Gupta, and M. Rees, "Patient 2.0 empowerment," in Proceedings of International Conference on Semantic Web and Web Services (SWWS '08), pp. 164-168, July 2008.

[18] R. Haase, T. Schultheiss, R. Kempcke, K. Thomas, and T. Ziemssen, "Use and acceptance of electronic communication by patients with multiple sclerosis: a multicenter questionnaire study," Journal of Medical Internet Research, vol. 14, no. 5, article e135, 2012.

[19] L. Fernandez-Luque, N. Elahi, and F. J. Grajales III, "An analysis of personal medical information disclosed in youtube videos created by patients with multiple sclerosis," in Proceedings of the 22nd International Conference on Medical Informatics Europe (MIE '09), pp. 292-296, September 2009.

[20] P. Wicks, M. Massagli, J. Frost et al., "Sharing health data for better outcomes on patientslikeme," Journal of Medical Internet Research, vol. 12, no. 2, 2010.

[21] C. A. Smith and P. J. Wicks, "PatientsLikeMe: consumer health vocabulary as a folksonomy," AMIA Annual Symposium Proceedings, pp. 682-686, 2008.

[22] A. Atreja, N. Mehta, D. Miller et al., "One size does not fit all: using qualitative methods to inform the development of an Internet portal for multiple sclerosis patients," AMIA Annual Symposium Proceedings, pp. 16-20, 2005.

[23] M. C. Hay, C. Strathmann, E. Lieber, K. Wick, and B. Giesser, "Why patients go online: multiple sclerosis, the internet, and physician-patient communication," Neurologist, vol. 14, no. 6, pp. 374-381, 2008.

[24] E. Murray, B. Lo, L. Pollack et al., "The impact of health information on the Internet on health care and the physician-patient relationship: National U.S. survey among 1.050 U.S. Physicians," Journal of Medical Internet Research, vol. 5, no. 3, article e17, pp. 38-53, 2003.

[25] I. Lejbkowicz, T. Paperna, N. Stein, S. Dishon, and A. Miller, "Internet usage by patients with multiple sclerosis: implications to participatory medicine and personalized healthcare," Multiple Sclerosis International, vol. 2010, Article ID 640749, 7 pages, 2010.

[26] R. A. Marrie, A. R. Salter, T. Tyry, R. J. Fox, and G. R. Cutter, "Preferred sources of health information in persons with multiple sclerosis: degree of trust and information sought," Journal of Medical Internet Research, vol. 15, no. 4, article e67, 2013.

[27] J. Wollin, H. Dale, N. Spencer, and A. Walsh, "The informational needs of the newly diagnosed person with multiple sclerosis and their family," International Journal of MS Care, vol. 2, no. 3, pp. $1-12,2000$.

[28] http://www.google.com/trends/.

[29] A. S. Khan, A. Fleischauer, J. Casani, and S. L. Groseclose, “The next public health revolution: public health information fusion and social networks," American Journal of Public Health, vol. 100, no. 7, pp. 1237-1242, 2010.

[30] M. Kang, H. Zhong, J. He et al., "Using Google trends for influenza surveillance in South China," PLoS ONE, vol. 8, no. 1, Article ID e55205, 2013. 
[31] F. Pervaiz, M. Pervaiz, N. Abdur Rehman, and U. Saif, "FluBreaks: early epidemic detection from Google flu trends," Journal of Medical Internet Research, vol. 14, no. 5, article e125, 2012.

[32] S. Cook, C. Conrad, A. L. Fowlkes, and M. H. Mohebbi, "Assessing Google flu trends performance in the United States during the 2009 influenza virus A (H1N1) pandemic," PLoS ONE, vol. 6, no. 8, Article ID e23610, 2011.

[33] J. Ginsberg, M. H. Mohebbi, R. S. Patel, L. Brammer, M. S. Smolinski, and L. Brilliant, "Detecting influenza epidemics using search engine query data," Nature, vol. 457, no. 7232, pp. 1012-1014, 2009.

[34] D. L. Heymann and L. Brilliant, "Surveillance in eradication and elimination of infectious diseases: a progression through the years," Vaccine, vol. 29, no. 4, pp. D141-D144, 2011.

[35] A. F. Dugas, Y.-H. Hsieh, S. R. Levin et al., "Google flu trends: correlation with emergency department influenza rates and crowding metrics," Clinical Infectious Diseases, vol. 54, no. 4, pp. 463-469, 2012.

[36] M. T. Malik, A. Gumel, L. H. Thompson, T. Strome, and S. M. Mahmud, “"Google flu trends” and emergency department triage data predicted the 2009 pandemic H1N1 waves in Manitoba," Canadian Journal of Public Health, vol. 102, no. 4, pp. 294297, 2011.

[37] V. M. Dukic, M. Z. David, and D. S. Lauderdale, "Internet queries and methicillin-resistant staphylococcus aureus surveillance," Emerging Infectious Diseases, vol. 17, no. 6, pp. 1068-1070, 2011.

[38] X. Zhou, J. Ye, and Y. Feng, "Tuberculosis surveillance by analyzing Google trends," IEEE Transactions on Bio-medical Engineering, vol. 58, no. 8, pp. 2247-2254, 2011.

[39] J. F. Gunn III and D. Lester, "Using Google searches on the internet to monitor suicidal behavior," Journal of Affective Disorders, vol. 148, no. 2-3, pp. 411-412, 2013.

[40] H. Sueki, "Does the volume of internet searches using suiciderelated search terms influence the suicide death rate: data from 2004 to 2009 in Japan," Psychiatry and Clinical Neurosciences, vol. 65, no. 4, pp. 392-394, 2011.

[41] A. C. Yang, S.-J. Tsai, N. E. Huang, and C.-K. Peng, "Association of Internet search trends with suicide death in Taipei City, Taiwan, 2004-009," Journal of Affective Disorders, vol. 132, no. 1-2, pp. 179-184, 2011.

[42] P. Deluca, Z. Davey, O. Corazza et al., "Identifying emerging trends in recreational drug use; outcomes from the Psychonaut Web Mapping Project," Progress in Neuro-Psychopharmacology and Biological Psychiatry, vol. 39, no. 2, pp. 221-226, 2012.

[43] P. M. Markey and C. N. Markey, "Seasonal variation in internet keyword searches: a proxy assessment of sex mating behaviors," Archives of Sexual Behavior, vol. 42, no. 4, pp. 515-521, 2013.

[44] N. F. Davis, L. G. Smyth, and H. D. Flood, "Detecting internet activity for erectile dysfunction using search engine query data in the Republic of Ireland," BJU International, vol. 110, no. 11, pp. E939-E942, 2012.

[45] S. D. Willard and M. M. Nguyen, "Internet search trends analysis tools can provide real-time data on kidney stone disease in the United States," Urology, vol. 81, no. 1, pp. 37-42, 2013.

[46] P. Wessa, "(Partial) Autocorrelation Function (v1.0.11) in Free Statistics Software (v1.1.23-r7)," Office for Research Development and Education, http://www.wessa.net/rwasp_autocorrelation.wasp/.
[47] C. Torrence and G. P. Compo, "A practical guide to wavelet analysis," Bulletin of the American Meteorological Society, vol. 79, no. 1, pp. 61-78, 1998.

[48] B. T. Grenfell, O. N. Bjørnstad, and J. Kappey, "Travelling waves and spatial hierarchies in measles epidemics," Nature, vol. 414, no. 6865, pp. 716-723, 2001.

[49] P. Wessa, "Multiple Regression (v1.0.26) in Free Statistics Software (v1.1.23-r7)," Office for Research Development and Education, http://www.wessa.net/rwasp_multipleregression.wasp/.

[50] P. Wessa, "Pearson Correlation (v1.0.6) in Free Statistics Software (v1.1.23-r7)," Office for Research Development and Education, http://www.wessa.net/rwasp_correlation.wasp/.

[51] A. V. Singh and P. Zamboni, "Anomalous venous blood flow and iron deposition in multiple sclerosis," Journal of Cerebral Blood Flow and Metabolism, vol. 29, no. 12, pp. 1867-1878, 2009.

[52] C. Vera, A. Herr, K. Mandato, M. Englander, L. Ginsburg, and G. P. Siskin, "Internet-based social networking and its role in the evolution of chronic cerebrospinal venous insufficiency," Techniques in Vascular and Interventional Radiology, vol. 15, no. 2, pp. 153-157, 2012.

[53] L. Machan, K. Murphy, and T. Traboulsee, "Multiple sclerosis and venous abnormalities: medicine in the age of social media," Canadian Association of Radiologists Journal, vol. 63, pp. S2-S4, 2012.

[54] J. W. Ayers, B. M. Althouse, J. P. Allem, J. N. Rosenquist, and D. E. Ford, "Seasonality in seeking mental health information on google," American Journal of Preventive Medicine, vol. 44, no. 5, pp. 520-525, 2013.

[55] M. Puthenparampil, D. Seppi, F. Rinaldi et al., "Increased incidence of multiple sclerosis in the Veneto region, Italy," Multiple Sclerosis, vol. 19, no. 5, pp. 601-604, 2013.

[56] A. Nicoletti, M. L. Lo Bartolo, S. Lo Fermo et al., "Prevelence and incidence of multiple sclerosis in Catania, Sicily," Neurology, vol. 56, no. 1, pp. 62-66, 2001.

[57] C. Solaro, C. Allemani, M. Messmer Uccelli et al., "The prevalence of multiple sclerosis in the north-west Italian province of Genoa," Journal of Neurology, vol. 252, no. 4, pp. 436-440, 2005.

[58] E. Granieri, C. Monaldini, R. De Gennaro et al., "Multiple sclerosis in the Republic of San Marino: a prevalence and incidence study," Multiple Sclerosis, vol. 14, no. 3, pp. 325-329, 2008.

[59] F. Ranzato, P. Perini, E. Tzintzeva et al., "Increasing frequency of multiple sclerosis in Padova, Italy: a 30 year epidemiological survey," Multiple Sclerosis, vol. 9, no. 4, pp. 387-392, 2003.

[60] M. Trojano, G. Lucchese, G. Graziano et al., "Geographical variations in sex ratio trends over time in multiple sclerosis," PLoS ONE, vol. 7, no. 10, Article ID e48078, 2012.

[61] E. Cocco, C. Sardu, R. Massa et al., "Epidemiology of multiple sclerosis in south-western Sardinia," Multiple Sclerosis, vol. 17, no. 11, pp. 1282-1289, 2011.

[62] M. Pugliatti, S. Sotgiu, G. Solinas et al., "Multiple sclerosis epidemiology in Sardinia: evidence for a true increasing risk," Acta Neurologica Scandinavica, vol. 103, no. 1, pp. 20-26, 2001.

[63] E. Millefiorini, A. Cortese, S. Di Rezze et al., "The prevalence of multiple sclerosis in central Italy," Multiple Sclerosis, vol. 16, no. 12, pp. 1432-1436, 2010.

[64] N. Koch-Henriksen and P. S. Sorensen, "Why does the northsouth gradient of incidence of multiple sclerosis seem to have disappeared on the Northern hemisphere?" Journal of the Neurological Sciences, vol. 311, no. 1-2, pp. 58-63, 2011.

[65] A. Lowe-Strong and P. J. McCullagh, "Monitoring of symptoms and interventions associated with multiple sclerosis," Studies in Health Technology and Informatics, vol. 117, pp. 223-228, 2005. 
[66] L. Wardell, A. M. Laizner, and Y. Lapierre, "Multiple sclerosis patients' interest in and likelihood of using online health-care services," International Journal of Multiple Sclerosis Care, vol. 11, no. 2, pp. 79-89, 2009.

[67] K. H. Jones, D. V. Ford, P. A. Jones et al., "The physical and psychological impact of multiple sclerosis using the MSIS-29 via the web portal of the UK MS Register," PLoS ONE, vol. 8, no. 1, Article ID e55422, 2013.

[68] R. E. Boeschoten, J. Dekker, B. M. Uitdehaag et al., "Internetbased self-help treatment for depression in multiple sclerosis: study protocol of a randomized controlled trial," BMC Psychiatry, vol. 12, p. 137, 2012. 


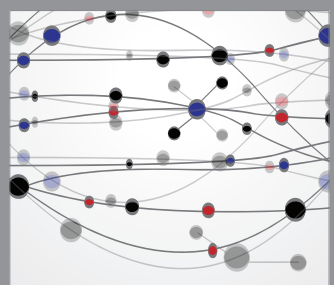

The Scientific World Journal
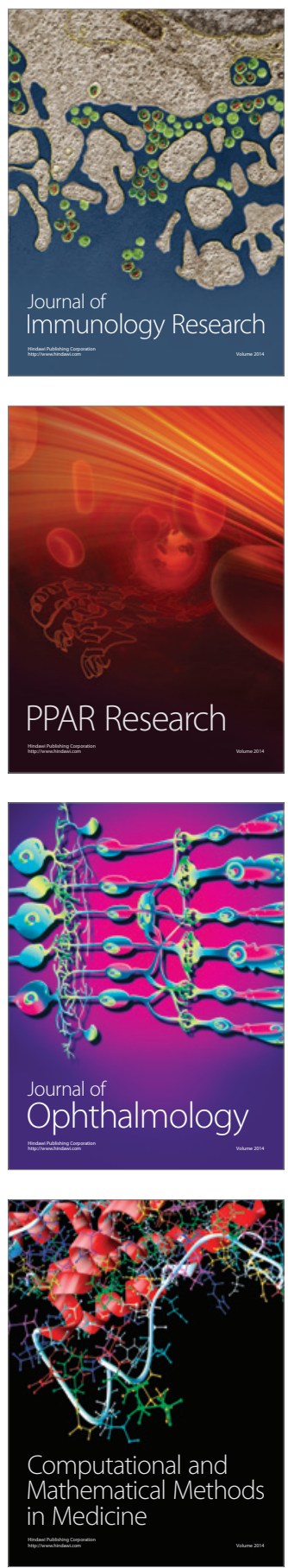

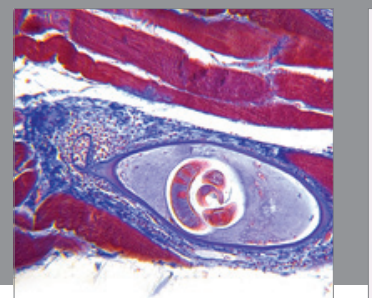

Gastroenterology

Research and Practice
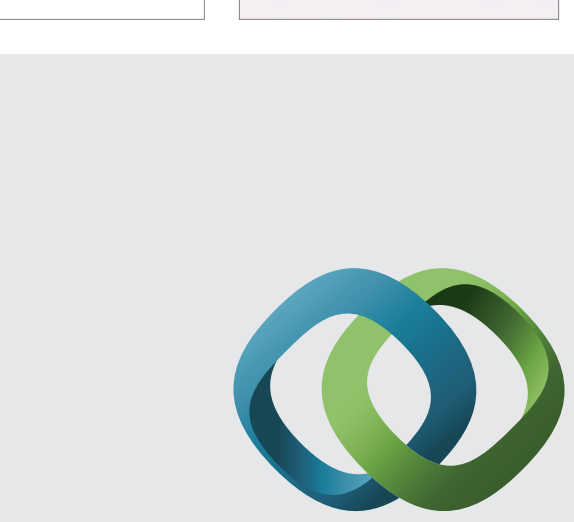

\section{Hindawi}

Submit your manuscripts at

http://www.hindawi.com
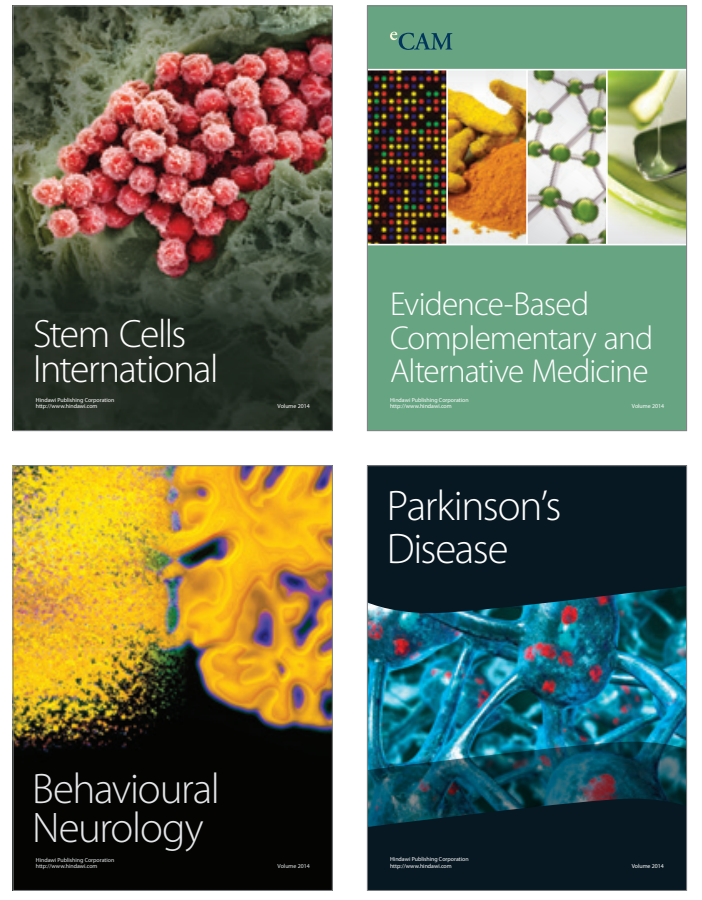
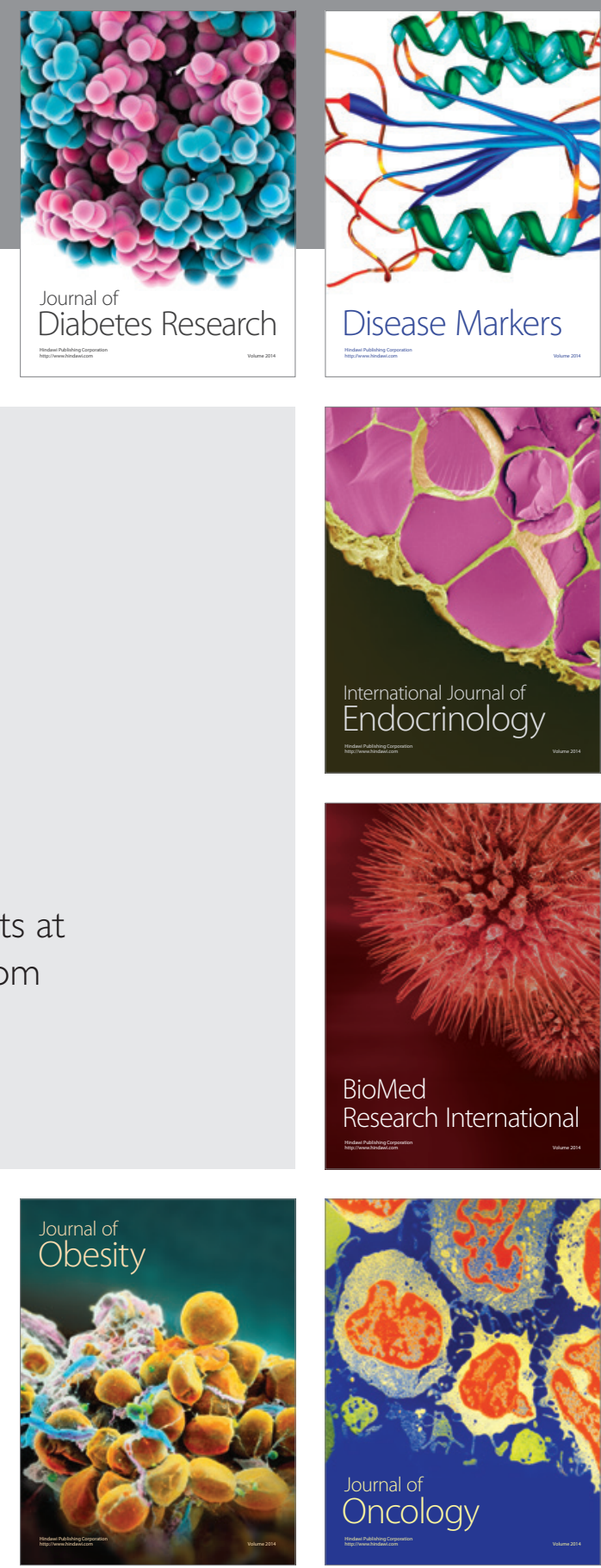

Disease Markers
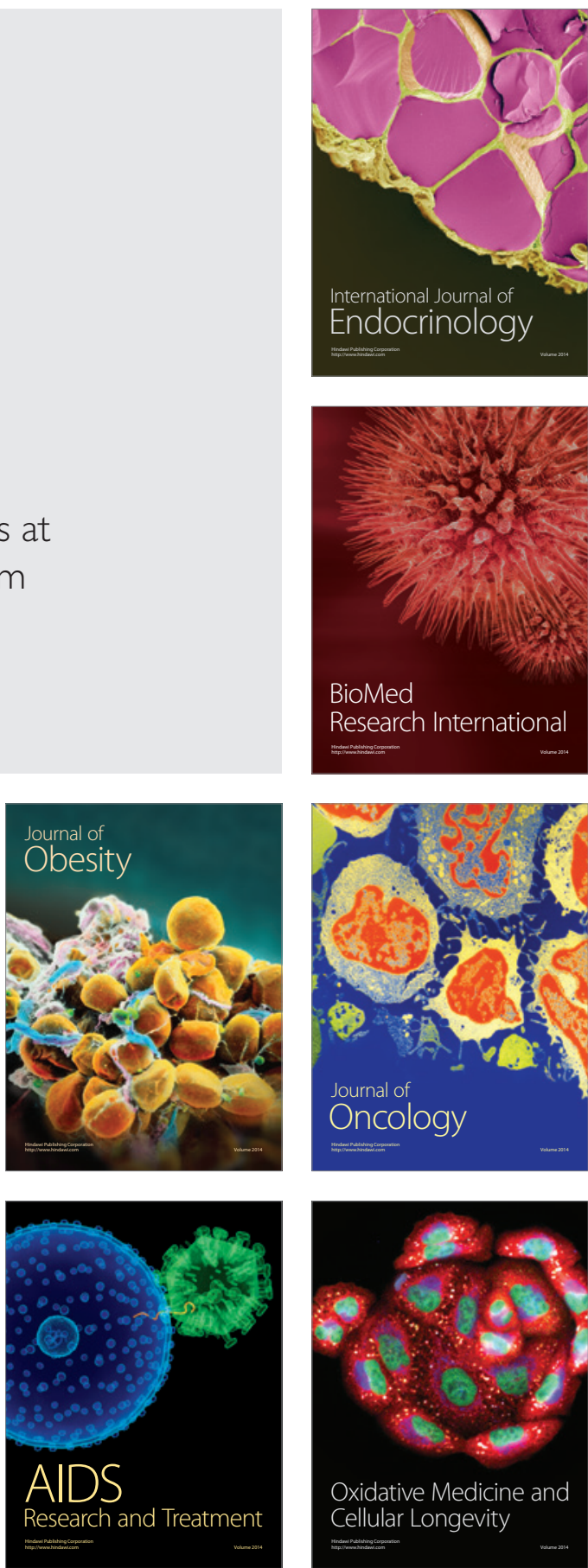